\title{
Expression of platelet-derived growth factors and their receptors in ovarian clear-cell carcinoma and its putative precursors
}

\author{
Sohei Yamamoto ${ }^{1}$, Hitoshi Tsuda ${ }^{1}$, Masashi Takano ${ }^{2}$, Tsunekazu Kita ${ }^{2}$, Kazuya Kudoh ${ }^{2}$, \\ Kenichi Furuya ${ }^{2}$, Seiichi Tamai ${ }^{3}$ and Osamu Matsubara ${ }^{1}$ \\ ${ }^{1}$ Department of Basic Pathology, National Defense Medical College, Tokorozawa, Saitama, Japan; \\ ${ }^{2}$ Department of Obstetrics and Gynecology, National Defense Medical College, Tokorozawa, Saitama, Japan \\ and ${ }^{3}$ Department of Laboratory Medicine, National Defense Medical College, Tokorozawa, Saitama, Japan
}

\begin{abstract}
Recent studies have shown that platelet-derived growth factors and their receptors are frequently co-expressed in ovarian cancers. Herein, we investigated the role of the platelet-derived growth factor pathway in the development of ovarian clear-cell adenocarcinoma, a highly chemoresistant form of ovarian cancer. Immunohistochemical expression of platelet-derived growth factor receptor- $\alpha$ and receptor- $\beta$, platelet-derived growth factor A-chain and B-chain was examined in 31 cases of clear-cell adenocarcinoma and 56 coexisting putative precursor lesions: 17 non-atypical and 19 atypical endometrioses, and 10 non-atypical and 10 atypical clear-cell adenofibroma components. Twenty-one solitary endometrioses were also examined. Vascular endothelial cells were always positive for all the markers examined, and were used as positive controls. The frequencies of positivity for platelet-derived growth factor receptor- $\alpha$ and receptor- $\beta$, and platelet-derived growth factor A-chain increased in accordance with higher cytologic atypia in the putative precursors: 71, 47, and $59 \%$ in the 17 non-atypical endometrioses, 84,73 , and $84 \%$ in the 19 atypical endometrioses, $0 \%$ each in the 10 non-atypical clear-cell adenofibromas, 100,90 , and $90 \%$ in the 10 atypical clear-cell adenofibromas, and $\mathbf{9 7}$, 97 , and $100 \%$ in the 31 clear-cell adenocarcinomas, respectively. Positivity for platelet-derived growth factor B-chain increased in accordance with increased atypia in clear-cell adenofibroma: $0 \%$ in non-atypical clear-cell adenofibromas, $30 \%$ in atypical clear-cell adenofibromas, and $60 \%$ in coexisting carcinomas. However, in contrast, positivity for platelet-derived growth factor B-chain decreased in accordance with increased atypia in endometriosis coexisting with clear-cell adenocarcinomas: $35 \%$ in non-atypical endometrioses, $11 \%$ in atypical endometrioses, and $5 \%$ in coexisting carcinomas. Platelet-derived growth factor receptor- $\alpha$ and receptor- $\beta$, and their ligands A-chain and B-chain were positive in 14, 29, 19, and $62 \%$ of the solitary endometrioses, respectively. These results indicate activation of the platelet-derived growth factor pathway in ovarian clear-cell adenocarcinomas and suggest biological differences between carcinomas that arise in association with clearcell adenofibroma vs endometriosis.
\end{abstract}

Modern Pathology (2008) 21, 115-124; doi:10.1038/modpathol.3800984; published online 14 December 2007

Keywords: ovarian clear-cell adenocarcinoma; PDGFR; autocrine/paracrine; endometriosis; clear-cell adenofibroma

Among the ovarian cancers, clear-cell adenocarcinoma has been recognized as a distinct clinicopathological entity because of its characteristic histology, frequent concurrence with endometriotic lesions, and highly chemoresistant nature resulting in an extremely poor prognosis when in high stage. ${ }^{1-3}$ Surgical debulking of the tumor is the only

Correspondence: Dr H Tsuda, MD, Department of Basic Pathology, National Defense Medical College, 3-2 Namiki, Tokorozawa, Saitama 359-8513, Japan.

E-mail: htsuda@ndmc.ac.jp

Received 19 June 2007; revised and accepted 1 October 2007; published online 14 December 2007 effective treatment for improving the prognosis of clear-cell adenocarcinoma. Although chemotherapeutic regimens including platinum analogues, taxanes, etoposide, and camptothecin have been developed, mortality of patients with ovarian clear-cell adenocarcinoma has remained largely unchanged. Therefore, more effective treatment options are needed, and these should be based on an understanding of the pathways driving neoplastic transformation and tumor growth. Some of these strategies may involve targeting of growth factors and growth factor receptors, which are important for the growth and development of specific cancer types. 
Despite a lack of data on cause-effect relationships, histological and epidemiological observations have consistently demonstrated a close association between endometriosis and ovarian clear-cell adenocarcinoma. ${ }^{1,4,5}$ In previous studies, loss of heterozygosity on chromosomes 9q, 10q, and 11q was detected in both clear-cell adenocarcinoma and coexisting endometriotic lesions. ${ }^{6,7}$ So far, however, there have been few data on the specific molecular or genetic alterations that are consistently involved in clear-cell adenocarcinoma, and it is still unclear whether a specific gene or chromosomal alteration is essential for clear-cell adenocarcinoma development.

On the other hand, clear-cell adenofibroma, a major form of benign or borderline ovarian clear-cell tumor, may be another type of clear-cell adenocarcinoma precursor. ${ }^{8,9}$ Our previous study demonstrated that clear-cell adenofibroma components coexisted in $21 \%$ of surgically resected clear-cell adenocarcinomas. ${ }^{10}$ These clear-cell adenofibroma components coexisting with clear-cell adenocarcinoma often contain both apparently benign clear-cell adenofibroma (clear-cell adenofibroma without atypia) and clear-cell adenofibroma with cellular and structural atypia (clear-cell adenofibroma with atypia or borderline clear-cell adenofibroma). ${ }^{8-10}$ Moreover, in comparison with clear-cell adenocarcinoma without clear-cell adenofibroma components, clear-cell adenocarcinoma with clear-cell adenofibroma components shows several distinct clinicopathologic characteristics, that is, a lower frequency of coexisting endometriosis, a higher frequency of histologically low-grade tumor with tubulocystic proliferative architecture, and lower cancer cell proliferative activity. ${ }^{10}$ These data suggest that, besides endometriosis, clear-cell adenofibroma components may be another form of clear-cell adenocarcinoma precursor, although there is no evident molecular background to support this speculation.

The platelet-derived growth factor receptors (PDGFRs: PDGFR- $\alpha$ and PDGFR- $\beta$ ) are transmembrane receptor tyrosine kinases that are activated by platelet-derived growth factors (PDGFs). ${ }^{11}$ The PDGFs are dimeric proteins composed of two closely related A-chain and B-chain polypeptides encoded by separate genes. The PDGFs selectively bind to receptor subunits via specific epitopes. PDGF-AA binds PDGFR- $\alpha$, whereas PDGF-AB and PDGF-BB recognize both PDGFR- $\alpha$ and PDGFR- $\beta .{ }^{11}$ On binding their ligands, the receptors dimerize and autophosphorylate specific tyrosine residues, resulting in activation of a variety of intracellular signaling molecules that control differentiation and cell proliferation. ${ }^{12}$ Tumor formation may result if these receptors are persistently activated through functional mutation or amplification of their genes, or if PDGFRs and PDGFs constitute autocrine/paracrine stimulating loops. Although such mutations and gene amplification have not been described pre- viously in ovarian cancers, recent studies have demonstrated that PDGFR- $\alpha$, PDGFR- $\beta$, and PDGF$\mathrm{AB}$ are commonly expressed in ovarian cancers at frequencies as high as 87,81 , and $67 \%$, respectively. ${ }^{13-16}$ Moreover, Henriksen et al ${ }^{17}$ have reported that PDGFRs or PDGFs were not detected in any of the benign ovarian tumors or normal ovarian epithelium they examined. Experiments in vivo have shown that PDGFRs activated by PDGFs modulate Akt and MAPK phosphorylation and significantly influence ovarian cancer cell proliferation and tumor expansion. ${ }^{16}$ These observations suggest that the PDGF-PDGFR system may play a functional role in the progression of ovarian cancers through autocrine or paracrine activation within the tumor tissues.

In the present study, we histologically reviewed a number of surgically resected cases of ovarian clearcell adenocarcinoma, and selected endometriotic lesions and clear-cell adenofibroma components synchronous with clear-cell adenocarcinoma. Using immunohistochemistry, we examined the expression of PDGFRs and PDGFs to clarify whether (1) PDGFRs and PDGFs are commonly expressed in clear-cell adenocarcinomas, (2) whether such expression is already evident in the two putative precursor forms of clear-cell adenocarcinoma, and (3) whether the expression status of these molecules is related to morphological changes in the epithelium of these putative precursors. It was anticipated that this information would not only lead to better understanding of the development of ovarian clearcell adenocarcinoma, but also provide insight into potentially promising treatment options for this highly chemoresistant malignancy.

\section{Materials and methods}

\section{Cases}

This study was performed with the approval of the Institutional Internal Review Board on ethical issues, and informed consent was obtained from all patients. Sixty-seven cases of primary ovarian clear-cell adenocarcinoma and 21 solitary endometriotic lesions were identified from the files of the Department of Laboratory Medicine, National Defense Medical College Hospital, Japan. These 67 cases of clear-cell adenocarcinoma had been surgically resected between the years 1987 and 2005, and the patients had not undergone chemotherapy or radiation therapy before surgery. Cases of solitary endometriosis were obtained by salpingo-oophorectomy with or without hysterectomy. All specimens were formalin-fixed and paraffin-embedded, and $4-\mu \mathrm{m}$ thick sections were prepared for hematoxylin and eosin staining. All pathology specimens were reviewed in our institution, and tumors were classified according to the criteria of the World Health Organization. ${ }^{8}$ 


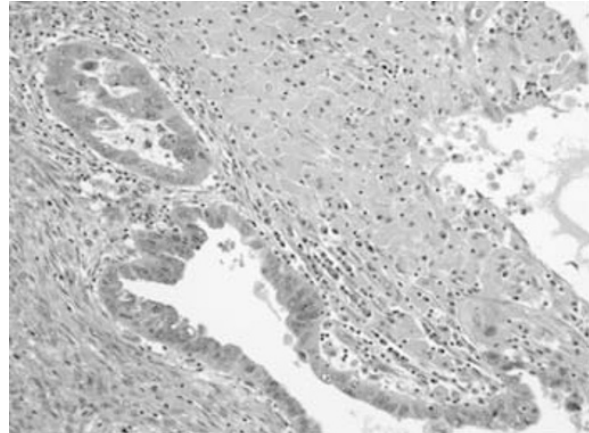

Figure 1 A case of endometriosis synchronous with clear-cell adenocarcinoma, showing cytologic aytpia. Nuclear enlargement and moderate pleomorphism are evident. H\&E staining, original magnification $\times 200$.

\section{Endometriosis Synchronous with Clear-Cell Adenocarcinoma}

Endometriosis synchronous with clear-cell adenocarcinoma was defined as (1) endometriosis existing in histological continuity with, or adjacent to, the clear-cell adenocarcinoma, or (2) an endometriotic cyst in which clear-cell adenocarcinoma was observed but histological continuity between the carcinoma and endometriotic epithelium was lacking.

With reference to the histological criteria of 'atypical endometriosis' described previously, cytological atypia was determined as present if, at most, one of the following features was histologically evident in the endometriotic epithelium: large hyperchromatic or pale nuclei with moderate to marked pleomorphism; an increased nuclear to cytoplasmic ratio; cellular crowding with stratification or tufting (Figure 1). ${ }^{5}$

Consequently, synchronous endometrioses were identified in 21 (31\%) of the 67 clear-cell adenocarcinomas. Of these 21, 15 (71\%) had both endometriotic lesions with and without cytologic atypia, 4 (19\%) had only endometriotic lesions with atypia, and $2(10 \%)$ had only endometriotic lesions without atypia. Therefore, 17 endometriotic lesions without atypia, 19 lesions with atypia, and 21 clearcell adenocarcinomas containing synchronous endometriosis were analyzed by immunohistochemistry.

\section{Clear-Cell Adenofibroma Components Synchronous with Clear-Cell Adenocarcinoma}

The histological criteria of clear-cell adenofibroma (with or without atypia) have been described previously. ${ }^{10}$ In short, clear-cell adenofibroma was a surface epithelial-stromal tumor containing tubulocystic epithelial components embedded in a fibroma-like stroma (Figure 2a). The epithelial cells were polygonal, hobnail or flat in shape, with clear, slightly granular or eosinophilic cytoplasm (Figure 2b). Presence of atypia was determined by histological documentation based on the cellular (ie, nuclear pleomorphism and stratification of the epithelium) and structural (ie, size irregularity and crowding of the tubulocystic architecture) features of each epithelial component (Figure 2c and d).

Consequently, of the 67 clear-cell adenocarcinomas, we identified $10(15 \%)$ cases that had both components of clear-cell adenofibroma with and without atypia. The designation 'clear-cell adenofibroma with atypia' is synonymous with, or has been referred to historically as clear-cell borderline tumor. $^{8,9}$ Therefore, 10 lesions of clear-cell adenofibroma without atypia, 10 lesions of clear-cell adenofibroma with atypia, and 10 clear-cell adenocarcinomas containing clear-cell adenofibroma components were analyzed by immunohistochemistry.

Among the 21 solitary endometrioses, reactive changes were identified in $6(29 \%)$ cases on the basis of the histological features described by La Grenade and Silverberg, ${ }^{18}$ that is, the epithelia had mildly hyperchromatic or pleomorphic nuclei, but lacked stratification, and were associated with moderate to severe subepithelial inflammation that is rarely seen in atypical endometriosis.

Presence or absence of atypia in endometriotic lesions and clear-cell adenofibroma components, and of reactive changes in solitary endometrioses were evaluated by two of the authors (SY and HT), and discussed until a consensus was reached. None of the 21 clear-cell adenocarcinomas with synchronous endometriosis and the 10 clear-cell adenocarcinomas with clear-cell adenofibroma components overlapped.

\section{Immunohistochemistry}

All selected formalin-fixed and paraffin-embedded specimens were cut into $4-\mu \mathrm{m}$ thick serial sections and analyzed by immunohistochemistry. We used rabbit polyclonal antibodies raised against a peptide mapped at the $\mathrm{C}$ terminus of PDGFR- $\alpha$ (RB-16981R7; prediluted; Neomarkers, CA, USA), PDGFR- $\beta$ (sc-339; dilution 1/100; Santa Cruz Biotechnology, Santa Cruz, CA, USA), and PDGF-A (sc-128; dilution 1/100; Santa Cruz Biotechnology), and against amino acids 136-190 of PDGF-B of human origin (sc-7878; dilution 1/100; Santa Cruz Biotechnology). Sections were deparaffinized and boiled in a microwave oven at $97^{\circ} \mathrm{C}$ for $20 \mathrm{~min}$ in $0.01 \mathrm{~mol} / \mathrm{l}$ citrate buffer (pH 6.0), then allowed to cool at room temperature. Endogenous peroxidase was blocked using $5 \%$ hydrogen peroxide. The slides were incubated at $4{ }^{\circ} \mathrm{C}$ overnight with primary antibodies and then reacted with a dextran polymer reagent combined with secondary antibodies and peroxidase (DAKO, Glostrup, Denmark) for $1 \mathrm{~h}$ at room temperature. Specific antigen-antibody reactions were visualized with $0.2 \%$ diaminobenzidine 

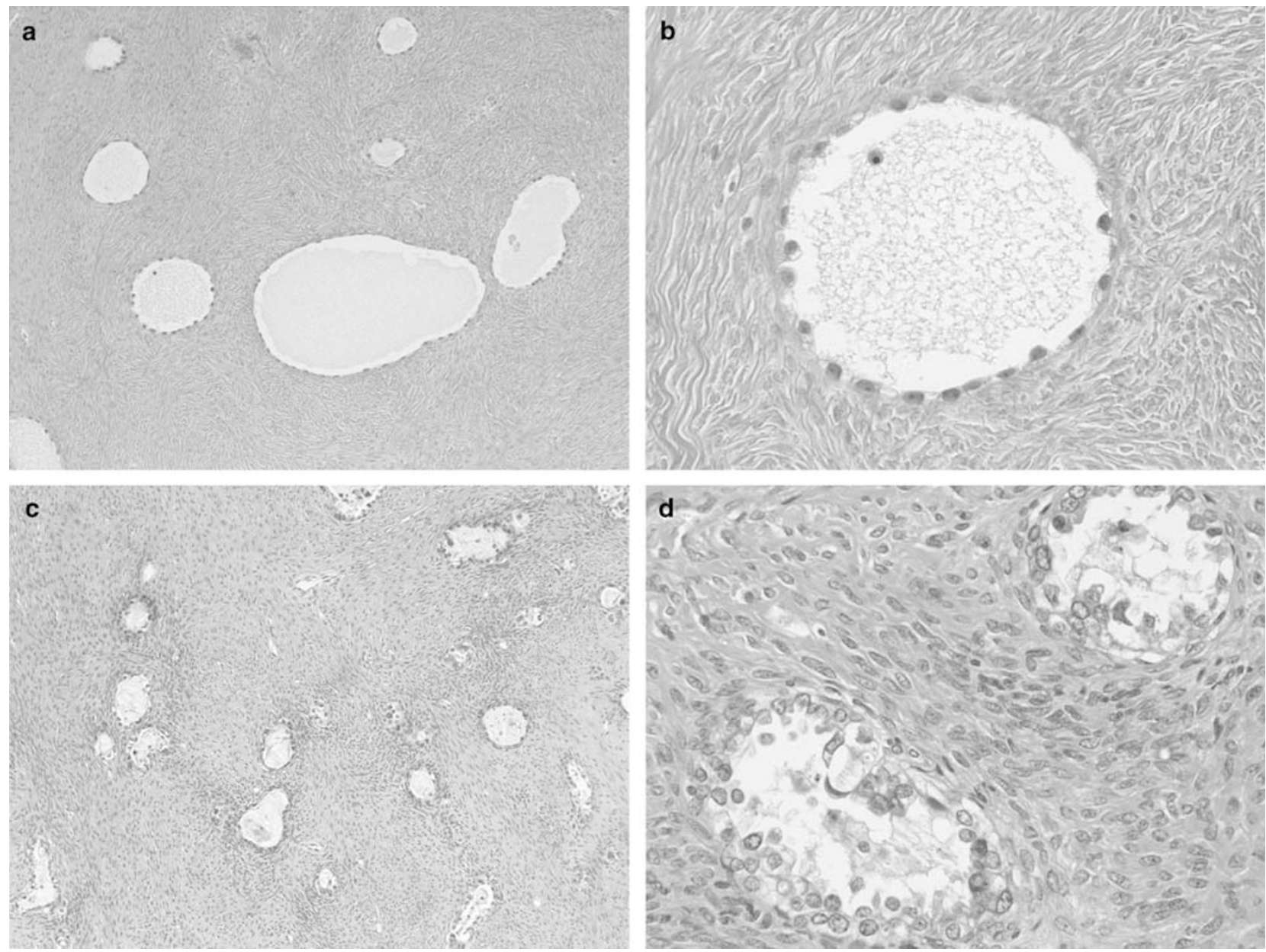

Figure 2 Histological features of clear-cell adenofibroma component without atypia (a and $\mathbf{b}$ ) and with atypia (c and d). (a) A clear-cell adenofibroma component shows simple cystic glands separated by wide fascicles of stromal cells. (b) Glands are delineated with monolayers of cuboidal, flattened, or in part hobnail glandular cells with clear cytoplasm and uniform nuclei. (c) Glands are variously sized and shaped, and focally crowded. (d) Glands are delineated with one to three layers of cells with clear to eosinophilic cytoplasm and mildly pleomorphic, hyperchromatic nuclei. H\&E staining, original magnification $\times 100$ for (a and $\mathbf{c})$; $\times 400$ for (b and d).

tetrahydrochloride and hydrogen peroxide, and counterstaining was performed using Mayer's hematoxylin. Fallopian tube epithelium or endothelium of vessels included in the sections served as a built-in-control for all four antibodies, and as a reference for normal expression of the proteins analyzed. ${ }^{19,20}$ As negative controls, sections without the primary antibody were used.

Cytoplasmic immunoreactivity for PDGFs (PDGF$A$ and PDGF-B) and both membranous and cytoplasmic immunoreactivity for PDGFRs (PDGFR- $\alpha$ and PDGFR- $\beta$ ) were taken into account for the evaluation. Immunoreactivity in the clear-cell adenocarcinomas was scored according to intensity as negative, weak $(1+)$, moderate $(2+$, similar to the control intensity), or strong $(3+)$. If $50 \%$ of epithelial components showed an immunoreactive intensity equal to or higher than that of fallopian tube epithelium, that is, $2+$ or $3+$, the cases (or lesions) were regarded as positive. Two observers (SY and HT) evaluated the results of the immunohistochemistry.

\section{Statistical Analyses}

Statistical analyses were performed using StatMate III software (ATMS, Tokyo, Japan). The frequency of immunopositivity for PDGFRs/PDGFs in the various components was compared using Fisher's exact test or $\chi^{2}$-test. Differences at $P<0.05$ were considered statistically significant.

\section{Results}

Results of immunohistochemistry are summarized in Tables 1 and 2.

\section{PDGFR/PDGF Expression in Clear-Cell Adenocarcinomas}

PDGFR- $\alpha$ expression was judged as positive in 30 $(97 \%)$ of the 31 clear-cell adenocarcinomas: 10 $(32 \%)$ cases scored $2+$ and $20(65 \%)$ scored $3+$ (Figure 3a). PDGFR- $\beta$ expression was positive in 30 
cases (97\%): $20(65 \%)$ cases scored $2+$ and 10 $(32 \%)$ scored $3+$ (Figure 3b). PDGF-A expression was positive in all 31 cases: $21(68 \%)$ cases scored $2+$ and $10(32 \%)$ scored $3+$ (Figure 3c). PDGFR- $\alpha$, PDGFR- $\beta$, and PDGF-A were positive in 95, 95, and $100 \%$ of the 21 clear-cell adenocarcinomas associated with endometriosis, and were always (100\%) positive in the 10 clear-cell adenocarcinomas with clear-cell adenofibroma components (Table 1).

PDGF-B was determined as positive in only 7 $(23 \%)$ cases, all of which scored $2+$ (Figure $3 \mathrm{~d}$ ) and showed co-positivity for PDGFR- $\beta$. Positivity for PDGF-B in clear-cell adenocarcinomas with clear-

Table 1 Immunoreactivity for PDGFRs and PDGFs in 31 cases of ovarian clear-cell adenocarcinoma

\begin{tabular}{|c|c|c|c|c|}
\hline \multirow[t]{3}{*}{ Molecules } & \multicolumn{4}{|c|}{$\begin{array}{l}\text { Number of cases (\%) } \\
\text { Immunoreaction }\end{array}$} \\
\hline & \multicolumn{2}{|c|}{ Negative } & \multicolumn{2}{|c|}{ Positive } \\
\hline & Score 0 & $1+$ & $2+$ & $3+$ \\
\hline PDGFR- $\alpha$ & 0 & 1 (3) & $10(32)$ & $20(65)$ \\
\hline PDGFR- $\beta$ & 0 & $1(3)$ & $20(65)$ & $10(32)$ \\
\hline PDGF-A & 0 & 0 & $21(68)$ & $10(32)$ \\
\hline PDGF-B & 9 (29) & $15(48)$ & $7(23)$ & 0 \\
\hline
\end{tabular}

PDGF, platelet-derived growth factor; PDGFR, platelet-derived growth factor receptor. cell adenofibroma components (6 of 10, 60\%) was significantly higher than that in clear-cell adenocarcinomas associated with endometriosis (1 of $21,5 \%$ ) $(P=0.0017)$ (Table 2).

\section{PDGFR/PDGF Expression in Endometriosis Synchronous with Clear-Cell Adenocarcinomas}

PDGFR- $\alpha$ and PDGFR- $\beta$ were positive in 12 (71\%) and $8(47 \%)$ of 17 endometriotic epithelia without cytologic atypia, respectively (Figure $4 \mathrm{a}$ and b). PDGF-A and PDGF-B were positive in 10 (59\%) and $6(35 \%)$ of the 17 endometriotic lesions without atypia, respectively (Figure 4c and $\mathrm{d}$ ), and all of these showed co-positivity with PDGFR- $\alpha$ and PDGFR- $\beta$, respectively (Table 2 ).

Among the 19 lesions showing cytologic atypia in the endometriotic epithelium, PDGFR- $\alpha$ and PDGFR- $\beta$ were positive in 16 (84\%) and 14 (73\%) cases, respectively (Figure $4 \mathrm{e}$ and f). PDGF-A and PDGF-B were positive in $16(84 \%)$ and $2(11 \%)$ of these 19 lesions (Figure 4g and h). Moreover, 15 (94\%) of 16 PDGF-A-positive lesions and 2 PDGF-Bpositive lesions showed co-positivity with PDGFR- $\alpha$ and PDGFR- $\beta$, respectively. Although PDGFRs (PDGFR- $\alpha$ and PDGFR- $\beta$ ) and PDGF-A tended to be more frequently positive in the endometriotic lesions with cytologic atypia than in those without, the difference was not statistically significant.

Table 2 Immunoreactivity for PDGFRs and PDGFs in clear-cell adenocarcinoma and in its putative precursor lesions

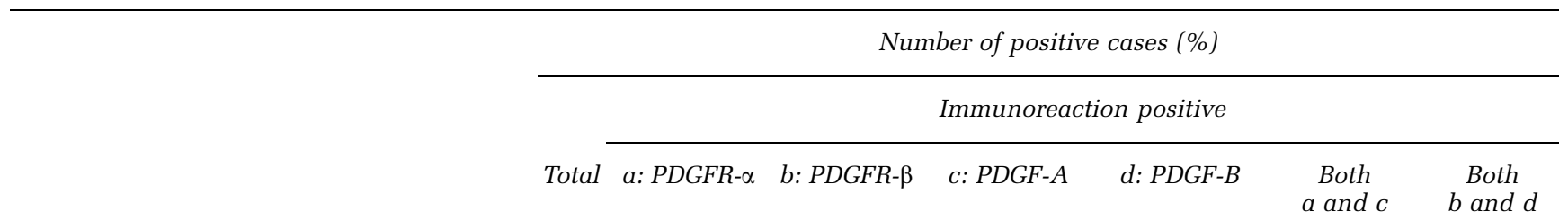

I: Immunoreaction in the putative precursors and clear-cell adenocarcinomas

\begin{tabular}{|c|c|c|c|c|c|c|c|c|c|c|}
\hline Endometriosis without atypia & 17 & $12(71)$ & 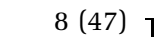 & $10(59)$ & & $6(35) 7$ & & $10(59) 7$ & & $6(35)$ \\
\hline Endometriosis with atypia & 19 & $16(84)$ & $14(73)$ & $16(84)$ & * & $2(11)$ & * & $15(79)$ & * & $2(11)$ \\
\hline Clear-cell adenocarcinoma & 21 & $20(95)$ & $20(95)$ & $21(100)$ & & $1(5)$ & & $20(95)$ & & $1(5)$ \\
\hline \multicolumn{11}{|c|}{ Clear-cell adenofibroma synchronous with clear-cell adenocarcinoma } \\
\hline Clear-cell adenofibroma without atypia & 10 & 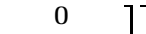 & 0 & $01]$ & & 0 & & $0]$ & & 0 \\
\hline Clear-cell adenofibroma with atypia & 10 & $10(100)\rfloor$ & $9(90)]$ & $9(90)\rfloor$ & * & $3(30)$ & * & $9(90)\rfloor$ & * & $3(30)$ \\
\hline Clear-cell adenocarcinoma & 10 & $10(100)$ & $10(100)$ & $10(100)$ & & $6(60)$ & & $10(100)$ & & $6(60)\rfloor$ \\
\hline Solitary endometriosis & 21 & $3(14)$ & $6(29)$ & $4(19)$ & & $13(62)$ & & $1(5)$ & & $5(24)$ \\
\hline Endometriosis without reactive change & 15 & $1(7)$ & $3(20)$ & $3(20)$ & & $7(47)$ & * & 0 & & $2(13)$ \\
\hline Endometriosis with reactive change & 6 & $2(33)$ & $3(50)$ & $1(17)$ & & $6(100)$ & & $1(17)$ & & $3(50)$ \\
\hline
\end{tabular}

II: Comparison of the PDGFRs and PDGF expression between solitary endometriosis and endometrioses synchronous with clear-cell adenocarcinomas

$\left.\left.\left.\begin{array}{lccc}\text { Solitary endometriosis } & 21 & 3(14) \\ \text { Endometriosis synchronous with clear-cell } & 17 & 12(71)\end{array}\right] * \begin{array}{ccc}6(29) & 4(19) \\ 8(47) & 10(59)\end{array}\right] * \begin{array}{ccc}13(62) & 1(5) \\ 6(35) & 10(59)\end{array}\right] * \begin{aligned} & 5(24) \\ & 6(35)\end{aligned}$

adenocarcinomas $^{\mathrm{a}}$

PDGF, platelet-derived growth factor; PDGFR, platelet-derived growth factor receptor.

${ }^{*} P<0.05$.

${ }^{\mathrm{a}}$ Endometriosis without cytologic atypia. 

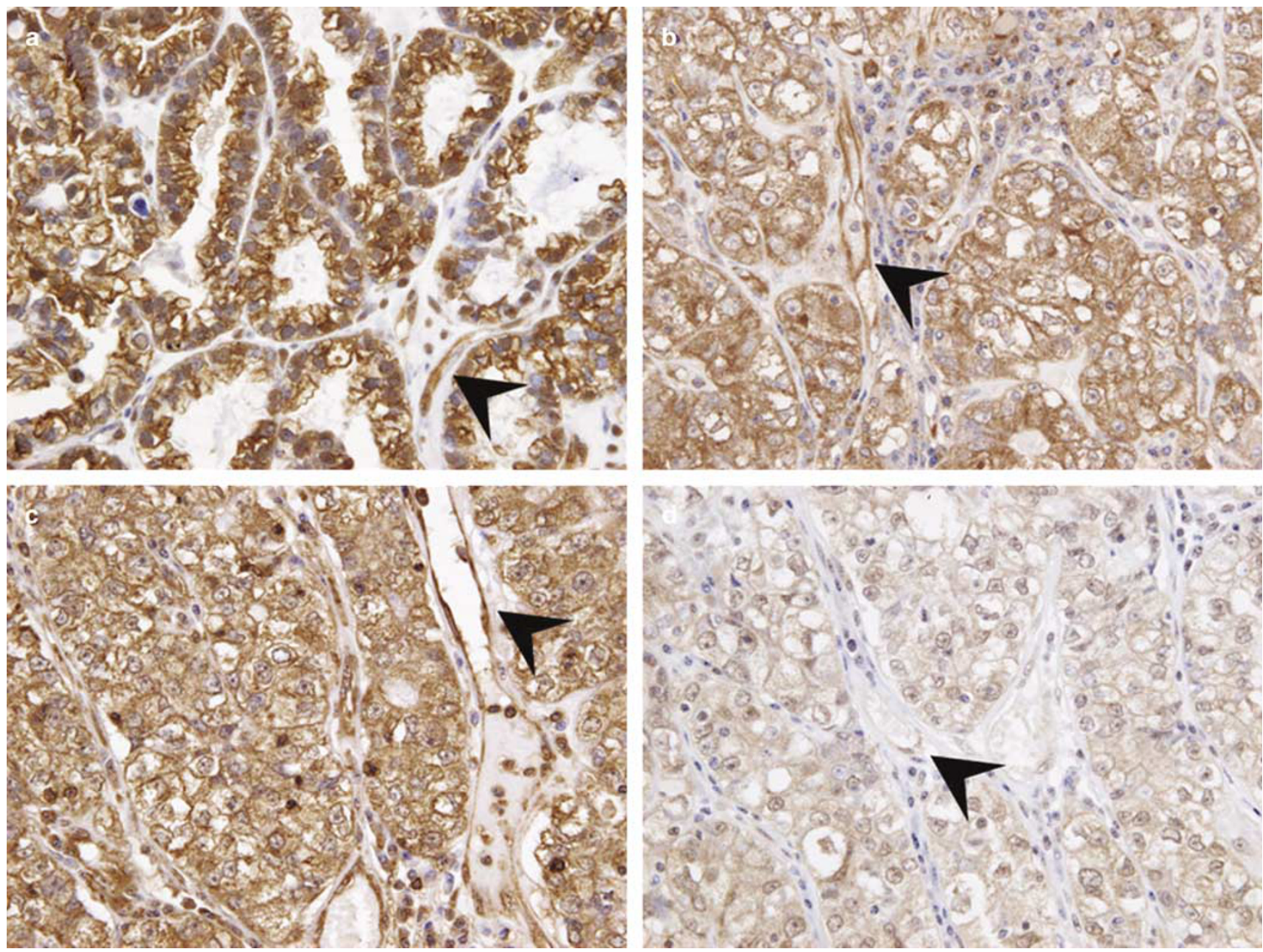

Figure 3 Expression of PDGFR- $\alpha$, PDGFR- $\beta$, PDGF-A, and PDGF-B in ovarian clear-cell adenocarcinomas. Positive immunoreactions for (a) PDGFR- $\alpha$ (scored $3+$ ), (b) PDGFR- $\beta$ (scored $3+$ ), (c) PDGF-A (scored $3+$ ), and (d) PDGF-B (scored $2+$ ). Arrowheads indicate the capillary endothelium showing moderate immunoreactivity with each primary antibody. Immunoperoxidase staining, original magnification $\times 400$.

Immunopositivity for PDGFR- $\alpha$, PDGFR- $\beta$, and PDGF-A in endometriotic lesions without atypia was lower than that in clear-cell adenocarcinomas: $71 \%$ (12 of 17) vs $95 \%$ (20 of 21 ), $47 \%$ ( 8 of 17) vs $95 \%$ (20 of 21 ), and $59 \%$ (10 of 17 ) vs $100 \%$ (21 of 21), respectively. In contrast, PDGF-B was expressed more frequently in synchronous endometriotic lesions without atypia than in clear-cell adenocarcinomas: $35 \%$ (6 of 17) vs 5\% (1 of 21), respectively (Table 2I). Consequently, comparison between endometriotic lesions without atypia and coexisting clear-cell adenocarcinomas revealed significant differences with regard to PDGFR- $\beta \quad(P=0.0011)$, PDGF-A $(P=0.0015)$, and PDGF-B $(P=0.022)$.

PDGFR/PDGF Expression in the Clear-Cell Adenofibromas Synchronous with Clear-Cell Adenocarcinomas

PDGFRs (PDGFR- $\alpha$ and PDGFR- $\beta$ ) and PDGFs (PDGF-A and PDGF-B) were negative in the epithe- lia of clear-cell adenofibroma components without atypia (Figure $4 \mathrm{i}-\mathrm{l}$ ) (Table 2 ).

Of the 10 clear-cell adenofibroma components with aytpia, PDGFR- $\alpha$ and PDGFR- $\beta$ were positive in $10(100 \%)$ and $9(90 \%)$ lesions, respectively (Figure $4 \mathrm{~m}$ and $\mathrm{n})$. PDGF-A and PDGF-B were positive in 9 $(90 \%)$ and $3(30 \%)$ of the 10 clear-cell adenofibroma components with atypia, respectively (Figure 4o and p). Moreover, all $9(100 \%)$ of the PDGF-A-positive lesions and all $3(100 \%)$ of the PDGF-B-positive lesions showed co-positivity with PDGFR- $\alpha$ and PDGFR- $\beta$, respectively.

\section{PDGFR/PDGF Expression in Solitary Endometriotic Epithelium}

Among the 21 cases with solitary endometriosis, PDGFR- $\alpha$, PDGFR- $\beta$, PDGF-A, and PDGF-B were positive in $3(14 \%), 6(29 \%), 4(19 \%)$, and 13 $(62 \%)$, respectively. One $(25 \%)$ of the 4 PDGF-Apositive cases and $5(42 \%)$ of the 13 PDGF-B- 

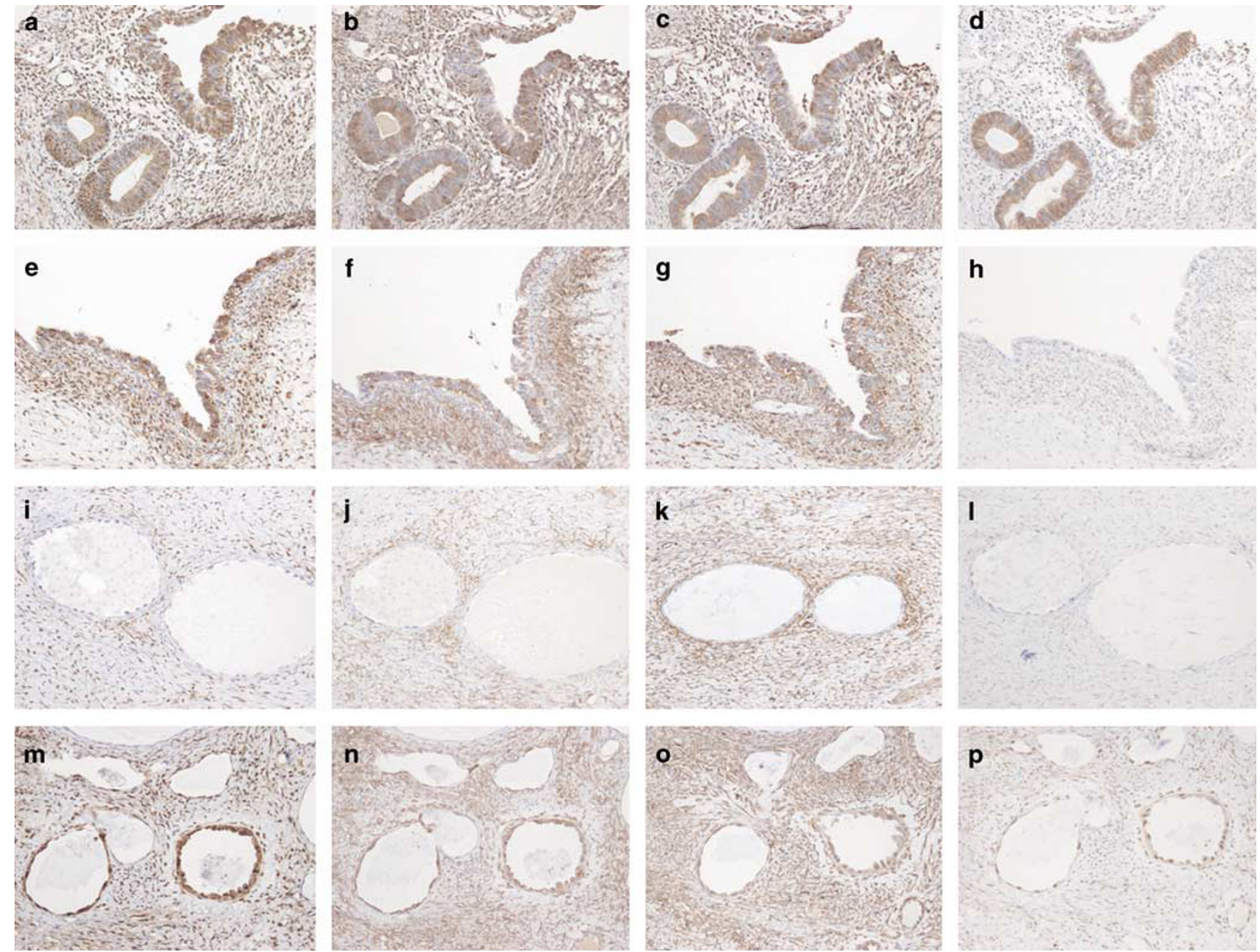

Figure 4 Expression of PDGFR- $\alpha$, PDGFR- $\beta$, PDGF-A, and PDGF-B in putative precursor lesions for ovarian clear-cell adenocarcinomas. Immunohistochemistry for PDGFR- $\alpha(\mathbf{a}, \mathbf{e}, \mathbf{i}$, and $\mathbf{m})$, PDGFR- $\beta(\mathbf{b}, \mathbf{f}, \mathbf{j}$, and $\mathbf{n})$, PDGF-A (c, $\mathbf{g}, \mathbf{k}$, and $\mathbf{o})$, and PDGF-B (d, h, l, and $\mathbf{p})$ in clearcell adenocarcinoma-associated endometriosis without cytologic atypia (a-d) and with cytologic atypia (e-h), clear-cell adenocarcinomaassociated clear-cell adenofibroma components without atypia (i-l), and with atypia ( $\mathbf{m}-\mathbf{p})$. PDGFR- $\alpha$, PDGFR- $\beta$, and PDGF-A are positive in the epithelial components of endometriosis without atypia (a-c) and with atypia $(\mathbf{e}, \mathbf{f}-\mathbf{g})$, and in clear-cell adenofibroma with atypia $(\mathbf{m}-\mathbf{o})$. PDGF-B is positive in epithelial components of endometriosis without atypia (d) and clear-cell adenofibroma with atypia (p), but negative in endometriosis with atypia (h), and clear-cell adenofibroma without atypia (l). Immunoperoxidase staining, original magnification $\times 200$.

positive cases showed co-positivity with PDGFR- $\alpha$ and PDGFR- $\beta$, respectively (Table 2 ).

Of the six lesions showing reactive changes, PDGFR- $\alpha$, PDGFR- $\beta$, PDGF-A, and PDGF-B were positive in two $(33 \%)$, three $(50 \%)$, one $(17 \%)$, and six $(100 \%)$, respectively (Figure $5 \mathrm{a}$ and b) (Table 2I). Therefore, PDGFRs and PDGF-B were more frequently positive in solitary endometrioses showing reactive changes than in those without, and there was a significant difference with regard to PDGF-Bpositivity ( $47 \%$ (7 of 15) vs $100 \%$ (6 of 6 ), $P=0.032$ ) (Table 2I).

When the solitary endometrioses and the endometrioses synchronous with clear-cell adenocarcinomas were compared, PDGFRs and PDGF-A were more frequently positive in the latter, and significant differences were evident with regard to PDGFR- $\alpha$ and PDGF-A positivity (PDGFR- $\alpha, 14 \%$ (3 of 21) in the former $v s 71 \%$ (12 of 17) in the latter, $P<0.001$; PDGF-A, 19\% (4 of 21) in the former vs $59 \%$ (10 of 17 ) in the latter, $P=0.014$ ) (Table 2II). On the other hand, PDGF-B was more frequently positive in solitary endometriosis than in endometriosis synchronous with clear-cell adenocarcinoma, but not to a significant degree (62\% (13 of 21) vs 35\% (6 of 17), $P=0.096)$ (Table 2II).

\section{Discussion}

In the present study, most $(97 \%, 30$ of 31$)$ of the ovarian clear-cell adenocarcinomas analyzed were positive for either PDGFR- $\alpha$ or PDGFR- $\beta$, and all of the 30 PDGFR- $\alpha$-positive carcinomas showed co-positivity for the PDGF-A chain, which is a component of PDGF-AA or PDGF-AB. Although several reports have documented the frequent 

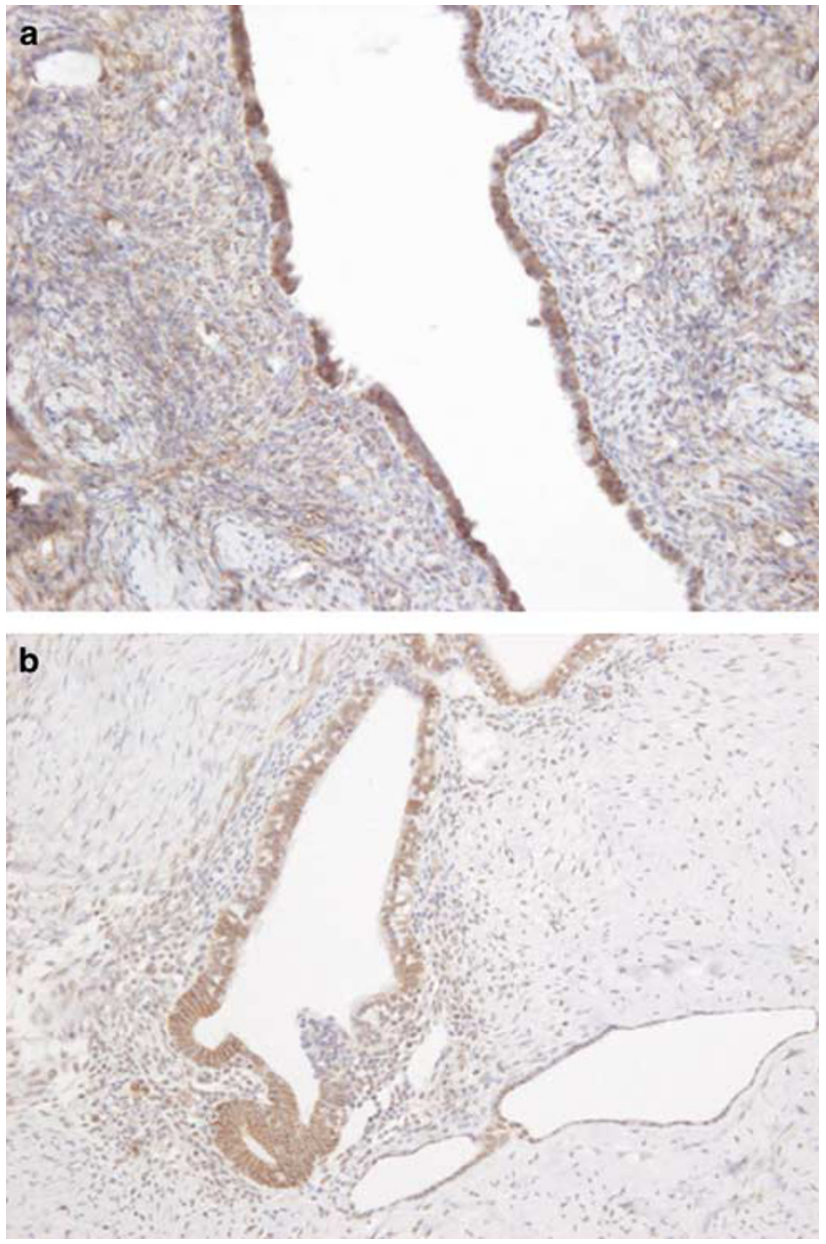

Figure 5 A case of solitary endometriosis showing positive immunoreactivity for (a) PDGFR- $\beta$ and (b) PDGF-B. Immunoperoxidase staining, original magnification $\times 200$.

expression of PDGFRs in ovarian carcinomas, the number of clear-cell adenocarcinoma cases included in those studies was generally small. ${ }^{13-15,17}$ The largest series by Matei et al ${ }^{16}$ demonstrated that 17 $(90 \%)$ and $18(95 \%)$ of 19 clear-cell adenocarcinomas were immunohistochemically positive for PDGFR- $\alpha$ and PDGF-AB, respectively, the former being almost concordant with our data.

In contrast, the frequency of positivity for the PDGF-B chain, which is a component of PDGF-AB or $-\mathrm{BB}$, was $23 \%$ of clear-cell adenocarcinomas in the present study, being much lower than the frequency of PDGF-AB expression (95\%) reported by Matei et al. ${ }^{16}$ This discrepancy may have resulted from the differences in the antibody used, protocols and evaluation of the immunohistochemistry. PDGFR- $\beta$, which is a receptor for PDGF-AB or PDGF-BB, was mostly (97\%) positive in the present series of clear-cell adenocarcinomas. However, PDGFs including PDGF-BB are natural ligands present in stromal cells such as fibroblasts, and the smooth muscle and endothelium of blood vessels, which constitute the major proportion of the stroma necessary for tumor growth and invasion, and these were constantly positive for PDGF-A and PDGF-B in the present study. ${ }^{19,20}$ These observations appear to support the hypothesis that not only an autocrine loop but also a paracrine loop of PDGFRs/PDGFs contributes to tumor progression of clear-cell adenocarcinoma. Although not studied in the present series, PDGF-D, which has been recently cloned, binds PDGFR- $\beta$ and shows mitogenic properties. ${ }^{21}$ The expression of PDGF-D in clear-cell adenocarcinoma would therefore be of interest.

In the endometriotic lesions, synchronous with clear-cell adenocarcinomas, the frequencies of PDGFR (PDGFR- $\alpha$ and PDGFR- $\beta$ ) and PDGF-A expression increased in accordance with the acquisition of cytologic atypia by the epithelium, and a similar trend was also evident in solitary endometriotic lesions. Moreover, except for PDGF-B, expression of PDGFs and PDGFRs was more frequent in endometriotic lesions synchronous with clearcell adenocarcinomas than in solitary endometrioses. These findings suggest that the establishment of autocrine/paracrine loops of PDGFRs/ PDGFs, especially those mediated by PDGFR- $\alpha$, might be an early event in the development of endometriosis-associated clear-cell adenocarcinomas, and that activation of PDGFR has already occurred by the stage of solitary endometriosis without cellular atypia.

In contrast to the endometriotic lesions, PDGFs and PDGFRs were not detected in the epithelium of apparently benign clear-cell adenofibroma components (clear-cell adenofibroma without atypia). However, similarly to endometriotic epithelium, expression of PDGFs and PDGFRs was frequently detected in clear-cell adenofibroma components when cytologic atypia was present. These findings suggest that establishment of autocrine/paracrine loops for PDGFR activation is not essential for the formation of clear-cell adenofibroma without atypia, but is highly associated with the acquisition of cytologic atypia by the epithelium of clear-cell adenofibroma and the development of clear-cell adenofibroma-associated clear-cell adenocarcinomas.

Our previous study demonstrated that clear-cell adenocarcinomas with clear-cell adenofibroma components were more frequently low-grade histologically, showed a tubulocystic architectural pattern, and had lower cancer cell proliferation activity than clear-cell adenocarcinomas without clear-cell adenofibroma, which were mostly $(68 \%)$ endometriosis-associated..$^{10}$ In the present study, the incidence of PDGF-B positivity was higher in clear-cell adenofibroma-associated clear-cell adenocarcinomas $(60 \%)$ than in endometriosis-associated clear-cell adenocarcinomas (5\%). All non-atypical clear-cell adenofibroma components studied were negative for PDGF-B. In contrast, PDGF-B positivity was relatively high in both solitary endometrioses $(62 \%)$ and non-atypical endometrioses synchronous with clear-cell adenocarcinoma (35\%). Therefore, it 
can be speculated that the role of PDGFR- $\beta$ and PDGF$\mathrm{BB}$ (or PDGF-AB) expression may be associated with cell differentiation rather than cell proliferation or neoplastic transformation, although how the difference in PDGF-B expression status between these clear-cell adenocarcinomas contributes to the biological nature of the tumors is largely unknown.

Ovarian carcinoma is one of the human epithelial malignancies showing histological heterogeneity, and clear-cell adenocarcinoma is one of its representative variations. ${ }^{1}$ Therefore, the possibility that areas of apparently benign endometriosis-like or clear-cell adenofibroma-like lesions represent well differentiated or 'mature' components of the carcinoma cannot be ruled out, although previous reports have indicated that stromal features of clear-cell adenofibroma (fibroma-like) could be an aid for differentiating clear-cell adenofibroma from clearcell adenocarcinoma with a prominent tubulocystic pattern. ${ }^{9,10}$ In the present study, expression of PDGF$\mathrm{A}$ and PDGFRs was detected less frequently in the endometriotic epithelium than in clear-cell adenocarcinomas, and was not detected in apparently benign clear-cell adenofibroma components. These observations suggest that the apparently benign areas coexisting with clear-cell adenocarcinomas, that is, non-atypical endometriosis and clear-cell adenofibroma without atypia, may be true precursors of ovarian clear-cell adenocarcinomas. Moreover, the striking differences in the frequency of expression of PDGFRs/PDGFs between endometriotic lesions and non-atypical clear-cell adenofibromas support the idea that clear-cell adenofibroma and endometriosis are two distinct forms of precursor for ovarian clear-cell adenocarcinoma.

The incidence of frequent high-intensity expression of PDGFRs in clear-cell adenocarcinomas demonstrated in this series appears to throw light on the molecular background involved in clear-cell adenocarcinoma formation, and also provides new insights into possible molecular-based therapies for this highly chemoresistant malignancy. PDGFRs, as well as KIT, belong to the type III receptor tyrosine kinases, and can be specifically treated by tyrosine kinase inhibitors such as imatinib mesylate (STI571). ${ }^{22}$ A recent study has demonstrated that imatinib mesylate inhibits ovarian cancer cell proliferation and PDGF-induced S-phase entry through PDGFR- $\alpha$ and Akt inactivation. ${ }^{23}$ Although a phase II trial of imatinib mesylate as a single agent failed to produce satisfactory efficacy against recurrent and platinum-resistant ovarian cancer, $^{24}$ cases with clear-cell adenocarcinoma were not enrolled and, therefore, the effect of imatinib or other tyrosine kinase inhibitors on ovarian clear-cell adenocarcinoma remains undetermined.

In the present study, we have demonstrated that PDGFRs and the PDGF-A chain are frequently expressed in ovarian clear-cell adenocarcinomas. Epithelial-epithelial and epithelial-stromal interactions via autocrine and/or paracrine activation of
PDGFRs might drive the development of clear-cell adenocarcinoma during the process of multistage carcinogenesis. The present results also suggest biological differences between clear-cell adenocarcinomas that arise in association with clear-cell adenofibroma vs endometriosis.

\section{Acknowledgements}

This work was supported in part by a grant-in-aid for special research from National Defense Medical College, and by a grant-in-aid for cancer research from the Ministry of Health, Labor, and Welfare, Japan. We are thankful to Dr David Douglas (Douglas' Scientific Editorial Services, Tokorozawa, Japan) for his professional review of the final version of the manuscript.

\section{Disclosure/conflict of interest}

We indicate no potential conflicts of interest.

\section{References}

1 Seidman JD, Russell P, Kurman RJ. Surface epithelial tumors of the ovary. In: Kurman RJ (ed). Blaustein's Pathology of the Female Genital Tract, 5th edn. Springer-Verlag: New York, 2001, pp 791-904.

2 Sugiyama T, Kamura T, Kigawa J, et al. Clinical characteristics of clear cell carcinoma of the ovary: a distinct histologic type with poor prognosis and resistance to platinum-based chemotherapy. Cancer 2000;88:2584-2589.

3 Ikeda K, Sakai K, Yamamoto R, et al. Multivariate analysis for prognostic significance of histologic subtype, GST-pi, MDR-1, and p53 in stages II-IV ovarian cancer. Int J Gynecol Cancer 2003;13:776-784.

4 Vercellini P, Parazzini F, Bolis G, et al. Endometriosis and ovarian cancer. Am J Obstet Gynecol 1993;169: 181-182.

5 Fukunaga M, Nomura K, Ishikawa E, et al. Ovarian atypical endometriosis: its close association with malignant epithelial tumours. Histopathology 1997;30: 249-255.

6 Jiang X, Morland SJ, Hitchcock A, et al. Allelotyping of endometriosis with adjacent ovarian carcinoma reveals evidence of a common lineage. Cancer Res 1998;58: 1707-1712.

7 Sato N, Tsunoda H, Nishida M, et al. Loss of heterozygosity on 10q23.3 and mutation of the tumor suppressor gene PTEN in benign endometrial cyst of the ovary: possible sequence progression from benign endometrial cyst to endometrioid carcinoma and clear cell carcinoma of the ovary. Cancer Res 2000;60: 7052-7056.

8 Tavassoli FA, Devilee P, (eds). World Health Organization classification of tumours. Pathology and Genetics of Tumours of the Breast and Female Genital Organs. IARC Press: Lyon, 2003, pp 218-228.

9 Bell DA, Scully RE. Benign and borderline clear cell adenofibromas of the ovary. Cancer 1985;56: 2922-2931. 
10 Yamamoto S, Tsuda H, Yoshikawa T, et al. Clear cell adenocarcinoma associated with clear cell adenofibromatous components: a subgroup of ovarian clear cell adenocarcinoma with distinct clinicopathological characteristics. Am J Surg Pathol 2007;31:999-1006.

11 Hart CE, Forstrom JW, Kelly JD, et al. Two classes of PDGF receptor recognize different isoforms of PDGF. Science 1988;240:1529-1531.

12 Heldin CH, Ostman A, Ronnstrand L. Signal transduction via platelet-derived growth factor receptors. Biochim Biophys Acta 1998;1378:F79-F113.

13 Schmandt RE, Broaddus R, Lu KH, et al. Expression of c-ABL, c-KIT, and platelet-derived growth factor receptor-beta in ovarian serous carcinoma and normal ovarian surface epithelium. Cancer 2003;98: 758-764.

14 Lassus H, Sihto H, Leminen A, et al. Genetic alterations and protein expression of KIT and PDGFRA in serous ovarian carcinoma. Br J Cancer 2004;91: 2048-2055.

15 Wilczynski SP, Chen YY, Chen W, et al. Expression and mutational analysis of tyrosine kinase receptors c-kit, PDGFRalpha, and PDGFRbeta in ovarian cancers. Hum Pathol 2005;36:242-249.

16 Matei D, Emerson RE, Lai YC, et al. Autocrine activation of PDGFRalpha promotes the progression of ovarian cancer. Oncogene 2006;25:2060-2069.

17 Henriksen R, Funa K, Wilander E, et al. Expression and prognostic significance of platelet-derived growth factor and its receptors in epithelial ovarian neoplasms. Cancer Res 1993;53:4550-4554.
18 La Grenade A, Silverberg SG. Ovarian tumors associated with atypical endometriosis. Hum Pathol 1988;19:1080-1084.

19 Siegbahn A, Hammacher A, Westermark B, et al. Differential effects of the various isoforms of plateletderived growth factor on chemotaxis of fibroblasts, monocytes, and granulocytes. J Clin Invest 1990;85: 916-920.

20 Tallquist MD, Soriano P, Klinghoffer RA. Growth factor signaling pathways in vascular development. Oncogene 1999;18:7917-7932.

21 Lokker NA, Sullivan CM, Hollenbach SJ, et al. Plateletderived growth factor (PDGF) autocrine signaling regulates survival and mitogenic pathways in glioblastoma cells: evidence that the novel PDGF-C and PDGF$\mathrm{D}$ ligands may play a role in the development of brain tumors. Cancer Res 2002;62:3729-3735.

22 Heinrich MC, Blanke CD, Druker BJ, et al. Inhibition of KIT tyrosine kinase activity: a novel molecular approach to the treatment of KIT-positive malignancies. J Clin Oncol 2002;20:1692-1703.

23 Matei D, Chang DD, Jeng MH. Imatinib mesylate (Gleevec) inhibits ovarian cancer cell growth through a mechanism dependent on platelet-derived growth factor receptor alpha and Akt inactivation. Clin Cancer Res 2004;10:681-690.

24 Coleman RL, Broaddus RR, Bodurka DC, et al. Phase II trial of imatinib mesylate in patients with recurrent platinum- and taxane-resistant epithelial ovarian and primary peritoneal cancers. Gynecol Oncol 2006; 101:126-131. 